\title{
Article \\ Efficient Simulations of Propagating Flames and Fire Suppression Optimization Using Adaptive Mesh Refinement
}

\author{
Caelan Lapointe ${ }^{1}$, Nicholas T. Wimer ${ }^{1}$, Sam Simons-Wellin ${ }^{1}$, Jeffrey F. Glusman ${ }^{1}$, Gregory B. Rieker ${ }^{1}$, and Peter \\ E. Hamlington ${ }^{1}$ \\ 1 Paul M. Rady Department of Mechanical Engineering, University of Colorado, Boulder, CO 80309, USA \\ * Correspondence: caelan.lapointe@colorado.edu
}

\begin{abstract}
Fires are complex multi-physics problems that span wide spatial scale ranges. Capturing this complexity in computationally affordable numerical simulations for process studies and "outerloop" techniques (e.g., optimization and uncertainty quantification) is a fundamental challenge in reacting flow research. Further complications arise for propagating fires where a priori knowledge of the fire spread rate and direction is typically not available. In such cases, static mesh refinement at all possible fire locations is a computationally inefficient approach to bridging the wide range of spatial scales relevant to fire behavior. In the present study, we address this challenge by incorporating adaptive mesh refinement (AMR) in fireFoam, an OpenFOAM solver for simulations of complex fire phenomena involving pyrolyzing solid surfaces. The AMR functionality in the extended solver, called fireDyMFoam, is load balanced, models gas, solid, and liquid phases, and allows us to dynamically track regions of interest, thus avoiding inefficient over-resolution of areas far from a propagating flame. We demonstrate the AMR capability and computational efficiency for fire spread on vertical panels, showing that the AMR solver reproduces results obtained using much larger statically refined meshes, but at a substantially reduced computational cost. We then leverage AMR in an optimization framework for fire suppression based on the open-source Dakota toolkit, which is made more computationally tractable through the use of fireDyMFoam. The extension of fireFoam developed here thus enables the use of higher fidelity simulations in optimization problems for the suppression of fire spread in both built and natural environments.
\end{abstract}

Keywords: numerical simulations; adaptive mesh refinement; fire spread; optimization

check for updates

Citation: Lapointe, C.; Wimer, N.T.; Simons-Wellin, S.; Glusman, J.F.; Rieker, G.B.; Hamlington, P.E. Efficient Simulations of Propagating Flames. Preprints 2021, 1, 0 . https://doi.org/

Received:

Accepted:

Published:

Publisher's Note: MDPI stays neutral with regard to jurisdictional claims in published maps and institutional affiliations.

\section{Introduction}

Given the potential hazard and cost of experimental studies, particularly at large spatial scales, computational simulations are an attractive approach to studying fire behavior. However, computationally efficient, full-physics simulations that avoid empirical ad hoc models [1] are required to study fire dynamics over widely varying and realistic conditions, as well as to enable the reliable use of "outer-loop" techniques such as optimization (e.g., for mitigation and suppression efforts) and uncertainty quantification (e.g., for risk assessment).

Perhaps the greatest difficulty in performing such high-fidelity simulations is the wide spatio-temporal scale separation characteristic of fire spread problems. Disparate temporal scales, from relatively slow flow advection to fast chemical reactions, are often addressed by assuming infinitely fast chemistry. This mixed-is-burnt assumption can be made because chemical reactions occur on much shorter timescales than the turbulent mixing timescale for non-premixed flames. The eddy dissipation model $[2,3]$ can then be used to relate the combustion timescale to local turbulence timescales. Flamelet models or other mechanism reduction approaches (e.g., at run-time [4-6] or a priori [7,8]) can also be used to balance fidelity with computational cost for problems where the mixed-is-burnt assumption is no longer valid.

Relevant spatial scales range from the small-scale structures of solid fuels to the larger scales characteristic of buildings and warehouses. Methods to address temporal scale separation generally increase the minimum computational timestep, either by not resolving 
the reaction timescale or by making finite-rate chemistry more affordable. However, the minimum spatial length scale is often dictated by geometric constraints, such as the fuel size or local geometry. Although static mesh refinement can be used to address some of this scale separation, such approaches are inadequate in fire spread problems where the region of interest may be rapidly varying and is not known a priori.

In the present study, we use adaptive mesh refinement (AMR) to address the challenge of bridging wide spatial scale separations in simulations of fire spread. Using AMR, grid cells and physical resolution - and, by extension, computational resources - can be concentrated where they are most needed, for example to track a propagating flame and make optimization and calibration studies (Nyazika et al. [9] provide a review) more tractable. Previously, Lapointe et al. [10] demonstrated the use of AMR with the OpenFOAM solver fireFoam [11] for simulations of turbulent diffusion flames, and here we introduce a new extension of this solver, called fireDyMFoam, that uses AMR to model fire spread over solid pyrolyzing surfaces. The new solver retains the pre-existing fireFoam capability for modeling multiphase flows, enabling the analysis and optimization of liquid-based fire suppression strategies (e.g., involving liquid films and Lagrangian particles) using computationally efficient AMR-based simulations of fire spread.

In the following, we outline the details of fireDyMFoam, including the treatment of gas, solid, and liquid phases, and provide a description of the AMR approach. We then demonstrate the solver for meter-scale vertical fire spread on combustible panels. We show, in particular, that the solver reproduces results from static mesh simulations with the same fine-scale resolution, but at a substantially reduced computational cost. We then leverage these computational savings and use fireDyMFoam inside an optimization loop, driven by the open-source Dakota toolkit for optimization and uncertainty quantification [12], to minimize fire spread on a combustible panel, similar to previous test cases examined using the Fire Dynamics Simulator [13-15]. The new solver also includes dynamic load balancing for efficient AMR of fire spread problems. We have made the solver freely available to the fire modeling community at the public GitHub repository https:/ / github.com/clapointe2 011/public for simulations and optimization of a broad range of fire spread problems.

\section{Computational framework: fireDyMFoam}

OpenFOAM is an open-source, finite-volume computational fluid dynamics platform that includes both pre- and post-processing functionalities, in addition to a wide array of solvers [16]. In the present study, we focus development on OpenFOAM-7 and implement AMR in the fireFoam solver $[10,11,17]$ for computationally efficient simulations of fire spread over solid pyrolyzing surfaces. Designed for simulations of industrial fire problems, fireFoam incorporates gas-, solid-, and liquid-phase regions, in addition to Lagrangian particle modeling capabilities. It has been used previously to study problems ranging from non-reacting buoyant plumes [18,19] and pool fires with static $[11,20,21]$ and dynamic meshes (i.e., using AMR) [10], to larger room-scale fires [22,23], pyrolysis modeling [24-28], and fire suppression $[26,29]$.

Here we extend the previous AMR solver outlined in Lapointe et al. [10] to address multiphase flows and solid fuel pyrolysis. The new solver, called fireDyMFoam, also includes dynamic load balancing to further increase computational efficiency. Each of these developments, which are described in more detail in the following sections, enable the fire suppression optimization framework described in Section 5.

\subsection{Physical treatment of the gas phase}

The fireFoam solver is typically used to perform large eddy simulations (LES), and this is also the approach that we take with the AMR-enabled solver fireDyMfoam. In the gas phase, three-dimensional (3D) Favre-filtered compressible Navier-Stokes equations 
and conservation equations for mass, total enthalpy, and species are solved on a single unstructured computational mesh. These equations are given as

$$
\begin{aligned}
\frac{\partial \rho}{\partial t}+\boldsymbol{\nabla} \cdot(\rho \boldsymbol{U}) & =S_{\mathrm{p}}^{(\rho)}+S_{\mathrm{f}}^{(\rho)}, \\
\frac{\partial(\rho \boldsymbol{U})}{\partial t}+\boldsymbol{\nabla} \cdot\left(\rho \boldsymbol{U}_{i} \boldsymbol{U}_{j}\right) & =-\boldsymbol{\nabla} p_{\mathrm{rgh}}+\boldsymbol{\nabla} \cdot \tau_{i j}+\rho \boldsymbol{g}+S_{\mathrm{p}}^{(U)} \\
\frac{\partial[\rho(h+K)]}{\partial t}+\boldsymbol{\nabla} \cdot[\rho \boldsymbol{U}(h+K)] & =\boldsymbol{\nabla} \cdot\left[\alpha_{\mathrm{eff}} \boldsymbol{\nabla}(\rho h)\right]+\frac{\partial p}{\partial t}+Q_{\mathrm{rxn}}+Q_{\mathrm{rad}}+S_{\mathrm{p}}^{(h)}+S_{\mathrm{f}}^{(h)}, \\
\frac{\partial\left(\rho Y_{i}\right)}{\partial t}+\boldsymbol{\nabla} \cdot\left(\rho \boldsymbol{U} Y_{i}\right) & =\boldsymbol{\nabla} \cdot\left[\alpha_{\mathrm{eff}} \boldsymbol{\nabla}\left(\rho Y_{i}\right)\right]+\rho \omega_{i}+S_{\mathrm{p}}^{\left(Y_{i}\right)}+S_{\mathrm{f}}^{\left(Y_{i}\right)},
\end{aligned}
$$

where unity Lewis and turbulent Prandtl numbers are assumed and $\tau_{i j}$ represents the viscous stress tensor given by

$$
\tau_{i j}=\nabla \cdot\left[\mu\left(\nabla \boldsymbol{U}+(\boldsymbol{\nabla} \boldsymbol{U})^{T}\right)-\lambda(\boldsymbol{\nabla} \cdot \boldsymbol{U}) \boldsymbol{I}\right] .
$$

In the above equations, $\rho$ is density, $\boldsymbol{U}$ is velocity, $p_{\text {rgh }}$ is dynamic pressure, $p$ is pressure (i.e., $p=p_{\text {rgh }}+\rho g \cdot x+p_{\text {ref }}$, where $p_{\text {ref }}$ is a reference pressure), $\boldsymbol{g}$ is gravitational acceleration, $h$ is specific sensible enthalpy, and $K$ is specific kinetic energy. The transport coefficients $\mu_{\text {eff }}$ and $\alpha_{\text {eff }}$ are the effective (i.e., molecular plus turbulent) viscosity and thermal diffusivity, respectively. In the enthalpy equation, $Q_{\mathrm{rxn}}$ and $Q_{\mathrm{rad}}$ represent energy sources (or sinks) due to reactions and radiation, respectively, and in the species transport equation $Y_{i}$ is the mass fraction of the $i^{\text {th }}$ species, and $\omega_{i}$ is the reaction rate for the $i^{t h}$ species. The terms $S_{\mathrm{p}}^{(\varphi)}$ and $S_{\mathrm{f}}^{(\varphi)}$ denote Lagrangian particle and liquid film source terms, respectively, for the variable $\varphi$, and are zero if unused; more detailed descriptions of these terms can be found in Wang et al. [11], Meredith et al. [26], Ren et al. [29] and Meredith et al. [30,31].

Depending on the choice of turbulence closure used to compute $\mu_{\text {eff }}$ and $\alpha_{\text {eff }}$, sub-grid scale (SGS) transport equations may also be necessary to close the governing equations. As in the standard version of fireFoam, we use mixture-averaged density and transport properties; the former is computed using the ideal gas law and the latter are temperaturedependent and computed using empirical correlations. Combustion modeling is assumed to be infinitely fast for non-premixed flames, although finite-rate multi-step chemical models can also be used, and governed by an eddy dissipation model. Radiation modeling is accomplished using a finite volume implementation of the discrete ordinate method, with a prescribed radiative fraction. For a detailed description of the gas phase physical treatment in fireFoam, we refer the interested reader to Wang et al. [11].

\subsection{Physical treatment of the solid phase}

In the solid phase, one-dimensional (1D) conservation equations are solved for mass, energy, and species, given as $[27,32,33]$

$$
\begin{aligned}
\frac{\partial \rho_{\mathrm{s}}}{\partial t} & =-R_{\text {gas }} \\
\frac{\partial\left(\rho_{\mathrm{s}} h_{\mathrm{s}}\right)}{\partial t}-\frac{\partial}{\partial x}\left[\frac{\partial\left(\kappa_{\mathrm{s}} T_{\mathrm{s}}\right)}{\partial x}\right] & =S_{\text {rad }}+S_{\mathrm{rxn}}+S_{\text {gas }}+S_{\text {flux }} \\
\frac{\partial\left(\rho_{\mathrm{s}} Y_{i, \mathrm{~s}}\right)}{\partial t} & =R_{i} .
\end{aligned}
$$

Here, $\rho_{\mathrm{s}}$ and $h_{\mathrm{s}}$ are solid-phase density and specific sensible enthalpy, $Y_{i, \mathrm{~s}}$ is the mass fraction of solid species $i, R_{i}$ is the reaction rate of the $i^{\text {th }}$ solid species, $R_{\text {gas }}$ is the corresponding rate of production of pyrolysate, and $\kappa_{\mathrm{S}}$ is the solid-phase thermal conductivity. Solid-phase energy source terms include an optional in-depth radiative source, $S_{\text {rad }}$, a reaction source term, $S_{\text {rxn }}$ a source term to account for mass loss (or, equivalently, gas production) from the solid phase, $S_{\text {gas }}$, and an optional enthalpy flux-based source term for 
gas motion within the solid, $S_{\text {flux }}$. These equations are solved on a series of independent, 1D solid meshes using mixture-averaged properties. That is, gas flux from the solid is computed from the solid mass loss rate and is assumed to immediately enter the gaseous region (or equivalently from pyrolysate production rate) [27,32,33]. Additional details of the solid phase modeling are available in Fukumoto et al. [27], Chaos et al. [32], and Vinayak [33].

\subsection{Physical treatment of liquid films and Lagrangian particles}

Liquid regions may be introduced at gas-solid interfaces, and are commonly used to "collect" water droplets for suppression modeling. If used, quasi-3D equations for mass, momentum, and energy conservation are solved in the liquid phase [26,29-31]. These equations are given by

$$
\begin{gathered}
\frac{\partial\left(\rho_{\mathrm{f}} \delta\right)}{\partial t}+\nabla_{\|} \cdot\left(\rho_{\mathrm{f}} \delta \boldsymbol{U}_{\|}\right)=S_{\rho_{\mathrm{f}},} \\
\frac{\partial\left(\rho_{\mathrm{f}} \delta \boldsymbol{U}_{\|}\right)}{\partial t}+\nabla_{\|} \cdot\left(\rho_{\mathrm{f}} \delta \boldsymbol{U}_{\|_{i}} \boldsymbol{U}_{\|_{j}}\right)=-\delta \boldsymbol{\nabla}_{\|} p_{\mathrm{f}}+\rho_{\mathrm{f}} \delta \boldsymbol{g}_{\|}+S_{U_{\|}}, \\
\frac{\partial\left(\rho_{\mathrm{f}} \delta h_{\mathrm{f}}\right)}{\partial t}+\nabla_{\|} \cdot\left(\rho \delta \boldsymbol{U}_{\|} h_{\mathrm{f}}\right)=S_{h_{\mathrm{f}}},
\end{gathered}
$$

where the $\|$ subscript denotes components tangential to the solid surface; vector terms therefore only have two components. For the film, $\rho_{\mathrm{f}}$ is the film density, $\delta$ is the film thickness, $U$ is the film velocity, $S_{\rho_{\mathrm{f}}}$ are mass source terms (e.g., addition due to coupling with Lagrangian particles, ejection of mass as Lagrangian particles, and phase change), $p_{\mathrm{f}}$ is the total film pressure (e.g., sum of local gas-phase, liquid hydrostatic and capillary pressure, and changes due to particle interaction), $s_{U_{\|}}$encompasses momentum-based source terms (e.g., viscous stresses and momentum addition or subtraction due to particle coupling), $g_{\|}$is the gravity vector, $h_{\mathrm{f}}$ is the film sensible enthalpy, and $S_{h_{\mathrm{f}}}$ are source terms in the energy equation (e.g., conductive and radiative heat transfer, phase change, and particle-based energy sources). A detailed description of all potential source terms is available in Meredith et al. [26], and a summary of film modeling as it is commonly used is available in Ren et al. [29]. The equations are solved on a two-dimensional (2D) mesh and the third direction is captured implicitly by the film thickness $\delta$.

If sprinklers are used (e.g., for flame suppression), a modeling methodology similar to other fireFoam sprinkler-suppression studies (e.g., Ren et al. [29] and FM Global [17]) is adopted such that water particles are only included as a mode of water delivery; heat transfer and phase change are not considered until the water droplets have been transferred to the solid panel surface and incorporated into the film region. Because the droplet mass, composition, and temperature remain constant, they are therefore governed only by a momentum conservation equation given as

$$
m \frac{\partial U_{\mathrm{p}}}{\partial t}=S_{U_{\mathrm{p}}}
$$

where $m$ is the particle mass, $\boldsymbol{U}_{\mathrm{p}}$ is its velocity and $\boldsymbol{S}_{U_{\mathrm{p}}}$ represents the forces acting on the particle. Fluid-particle coupling is two-way, and particle source terms are treated semi-implicitly.

\subsection{Phase coupling}

Gas-solid-liquid phase coupling is treated in a segregated manner; Lagrangian particles are solved first, followed by solution of the liquid, solid, and gas phases (if a phase is not required, its solution is simply skipped). Interactions between each phase are enabled by the various source terms in the transport equations described previously. At phase boundaries, the phases are coupled via mapped boundary conditions for the velocity, temperature, and reacting species. For velocity, the mass flux of pyrolysate in the solid 
region is matched to a corresponding mass flux of fuel in the gaseous region using either a one-to-one mapping or an energetically-equivalent mapping (e.g., as implemented in FM Global [17]). For temperature, heat transfer is coupled between the solid, liquid, and gaseous regions, and for the fuel, pyrolysate in the solid region is converted to a known fuel in the gaseous region.

\subsection{Adaptive mesh refinement (AMR)}

Previously, we developed an AMR solver for efficient simulations of turbulent diffusion flames, called diffusionFireFoam [10]. In the present work, we have extended the diffusionFireFoam solver to combine the OpenFOAM dynamic meshing functionality described in Jasak and Gosman [34] with the full suite of physical models (e.g., pyrolysis, Lagrangian particle, and surface film modeling) included in fireFoam. For the present work, AMR is constrained to the gas phase (where the solution is most computationally expensive); solid and liquid regions use static meshes. To simplify the use of AMR with mapped boundary conditions (which is necessary to couple solid, liquid, and gaseous regions), faces on any coupled boundary and, by extension, the cells containing said faces, are protected from refinement; they are pre-refined to the highest level of AMR that will be used.

The AMR implementation in OpenFOAM takes the form of a single, unstructured mesh, in contrast to block-structured AMR as used, for example, in Zhang et al. [35]. At user-specified intervals, the mesh is updated based on a single refinement field. Cells with values of the field between lower and upper bounds are marked for refinement, and are split in two in each coordinate direction, such that one cell becomes eight cells in 3D. Similarly, values of the field below a cutoff are identified for coarsening, where the reverse process occurs and cells are recombined. Other input parameters include the refinement frequency and a buffer parameter to "insulate" newly added cells (i.e., make the AMR cell addition more conservative).

The mesh update process is summarized by the following steps [10,34]

1. If the simulation timestep index is a multiple of a user-specified refinement interval, trigger the mesh update.

2. Scan the user-defined refinement field for cells eligible for refinement. Eligible cells are determined by comparing values of the field to lower and upper refinement bounds. If the cell value lies within the refinement bounds, it is marked as a candidate for refinement. Candidates are then scanned and checked for "refineability"; namely, candidates are considered refinable if they are below the max refinement level and if they are hexagonal cells.

3. Refine the selected cells and protect "buffer" cells around the newly refined cells from un-refinement. Map the solution to the new mesh.

4. Scan points in the mesh for un-refinement, comparing point values to an un-refine level; values lower than this level are tagged for recombination. Cells are then recombined and the solution is mapped once more.

We refer to Jasak and Gosman [34] and Lapointe et al. [10] for a more complete description of the AMR process, including verification and validation of the approach in a range of both non-reacting and reacting flow problems.

Previously, we extended the AMR library in Jasak and Gosman [34] to enable refinement and coarsening on an arbitrary number of fields [10]. These fields are scaled at run-time to range between 0 and 1 , although un-scaled fields may also be used; refinement bounds are therefore percents of the maximum value (e.g., the top $99.9 \%$ of values at a given time, or lower and upper bounds of 0.001 and 1, respectively). Prior work also involved extensive testing of the AMR procedure, focusing on flux corrections for new cell faces to ensure mass conservation. All of these improvements have also been implemented in fireDyMFoam.

Before continuing, it is prudent to discuss the application of AMR, specifically, within the context of fireFoam simulations. As described previously, fireFoam solves 3D conserva- 
tion equations only in the gas phase; solid and liquid phases are 1D and 2D, respectively. This is important for two reasons: (i) native AMR functionality is inherently $3 \mathrm{D}$ and (ii) phases are coupled with mapped boundary conditions (with an implied one-to-one mapping) at their respective interfaces.

With regard to the first point, we note that the majority of the computational expense can be attributed to the solution of the gas phase. Additionally, the solid and liquid phases use customized versions of "standard" OpenFOAM meshes, making integration of native AMR functionality non-trivial. Various researchers (e.g., Rettenmaier et al. [36]) have addressed this integration (e.g., by extending the AMR for use with meshes that are not 3D), which is beyond the scope of the current study.

Regarding the second point, extensions to support changing meshes at the interface would ideally be accompanied by the use of 3D meshes in the solid and liquid phases. This extension would also incorporate arbitrary mesh interface (AMI) functionality at interfaces; both extensions will be the focus of future work. For the current work, we focus on the use of AMR in the gas phase and make accommodations to facilitate its addition to fireFoam, such as pre-refining phase interfaces (to match the target maximum level of refinement) and protecting the interfaces from further refinement.

Recognizing that this pre-refinement (as well as the AMR itself) may introduce load imbalances for parallel simulations, we have also implemented run-time load balancing to maintain a balanced distribution of computational load for AMR cases run in parallel. This implementation follows that used in hyStrath [37], where processor imbalances are assessed at write-time and system calls are used to redistribute the computational mesh. This method is noteworthy because it allows for use of native OpenFOAM utilities (e.g., reconstructParMesh, reconstructPar, and decomposePar), but requires minimal code modification (mostly at the solver level) and is easily extendable to other solvers.

\subsection{Numerical treatment}

In the gas phase, the conservation equations are solved in a segregated manner and coupled using a procedure (i.e., PIMPLE) involving outer SIMPLE iterations and inner PISO correctors [11]. For this work, we have implemented a variant that places the energy and species conservation inside the PISO corrector loop, similar to implementations in other OpenFOAM solvers [38,39].

Spatial discretization is nominally second-order accurate; total variation diminishing schemes are employed for the divergence of conserved scalar quantities, and a stabilized central difference scheme is used for the velocity convective term to aid stability. Additionally, in the interest of stability, enthalpy gradients are limited. Diffusive terms are unbounded and second-order with limited non-orthogonal corrections. First-order Euler integration is used to march forward in time.

For the solid phase, we again use second-order methods for spatial discretization and first-order Euler integration for temporal integration. First-order integration in time is also used in the liquid phase, if present; spatially, divergence terms are also first-order and diffusive terms are second-order. A global timestep is computed from a user-specified maximum advective Courant number in the gaseous and film regions and maximum diffusion number in the solid region. Temporal sub-cycling is used (often with a lower, particle-specific Courant number) to march Lagrangian particles forward in time.

\section{Verification: Single panel vertical fire spread}

For the present implementation of AMR in fireDyMFoam to be deemed successful, it must result in a reduction of simulation cost with little or no impact on accuracy, as compared to equivalently refined static mesh simulations. To this end, we first compare the cost and accuracy of AMR in fireDyMFoam against a statically-refined simulation of a vertical fire spread problem (without water droplets or films, for simplicity).

The present case is based on a tutorial included with the standard distribution of OpenFOAM [16], where a $[0.3 \times 0.6] \mathrm{m}, 60 \mathrm{~kW}$ propane burner is placed in the center of a 
narrow, initially quiescent domain. The burner is bounded on two sides by $[0.6 \times 2.4 \times$ 0.01] m combustible wood panels, composed of homogeneous mixtures of unburnt solid fuel and burnt charred product. The propane burns infinitely fast as it mixes with ambient air, heating the wood. The wood pyrolyzes and combustible products are transferred to the gaseous region, where they burn and the flame spreads.

For this first demonstration of fireDyMFoam, we focus on a single panel for simplicity, as shown in Fig. 1. Static mesh and AMR simulations, described in the following, are performed for this simpler case, and the results are compared to verify that the AMR simulation produces comparable results to the static mesh simulation, but at a lower computational cost.

\subsection{Statically refined simulation}

In the static mesh simulation, a base mesh measuring $[0.8 \times 4.2 \times 1.2] \mathrm{m}$, centered on the propane burner, was created for the solution of the gaseous region with $20 \mathrm{~cm}$ resolution. Four nested, static regions of refinement were then created, including a finest refinement region with $1.25-\mathrm{cm}$ resolution occupying a $[0.4 \times 2.5 \times 0.8] \mathrm{m}$ volume centered longitudinally on the burner. Figures 2(a) and (d) show slices through the center of the resulting static mesh at $z=0$, indicating the successive regions of static refinement in the $x-y$ plane.

The solid panel region was created by extruding a collection of faces on the boundary of the base mesh, within the $1.25 \mathrm{~cm}$ resolution region. An area of size $[0.6 \times 2.4] \mathrm{m}$ was extruded by $1 \mathrm{~cm}$, with $1 \mathrm{~mm}$ resolution in the third dimension. The propane burner was created in a $[0.1 \times 0.4] \mathrm{m}$ area on the bottom boundary.

Gas and solid regions were coupled as described in Section 2.6. Within the gas phase, turbulence modeling was accomplished using a one equation eddy-viscosity model with $C_{k}=0.03$ [40]. Combustion was modeled using the eddy dissipation method, as implemented in FM Global [17], with coefficients $C_{\text {EDC }}, C_{\text {diff, }}$, and $C_{\text {stiff }}$ of $4.0,0.4$, and $10^{-10}$, respectively [41,42]. Radiation modeling was performed using the finite volume discrete ordinate method with a prescribed radiative fraction of 0.2 (similar to that used in Ren et al. [29]). In the solid phase, model coefficients for thermophysical properties match those from Chaos et al. [43] for the single-layer configuration. Temporal and spatial discretization is set as described in Section 2.6.

As mentioned previously, a custom variant of the PIMPLE algorithm is used [38,39]; one outer PIMPLE iteration (where mass, momentum, and turbulence are solved) and three inner PISO iterations (where species, energy conservation, and pressure correctors are solved) are used to solve the gas-phase conservation equations. A maximum convective Courant number of 0.5 is enforced in the gas phase, and a maximum diffusion number of 0.25 is used for the solid phase; the most limiting constraint is used to compute a single global timestep.

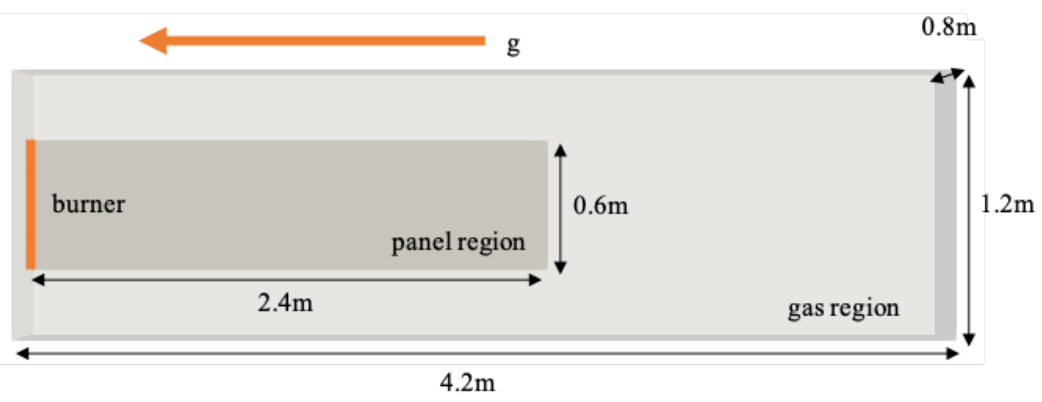

Figure 1. Schematic of the vertical fire spread case, showing the propane burner in orange, as well as both the panel and gas regions. The orange arrow indicates the direction of gravity. 

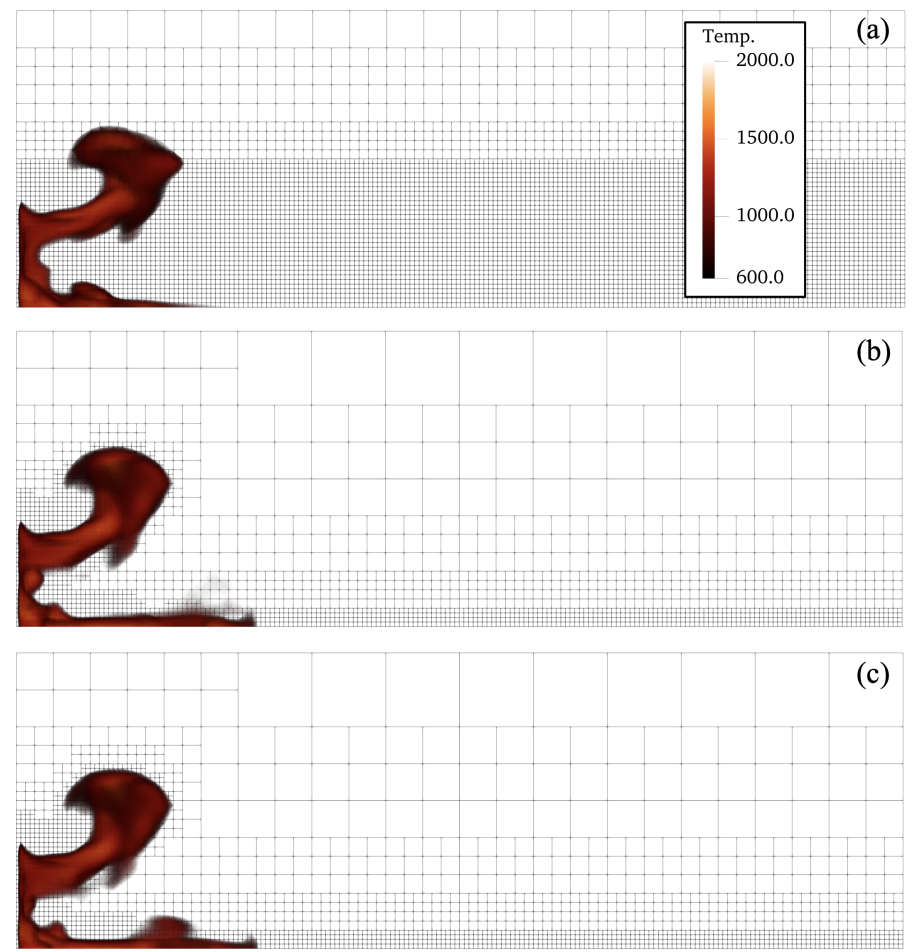

(a)

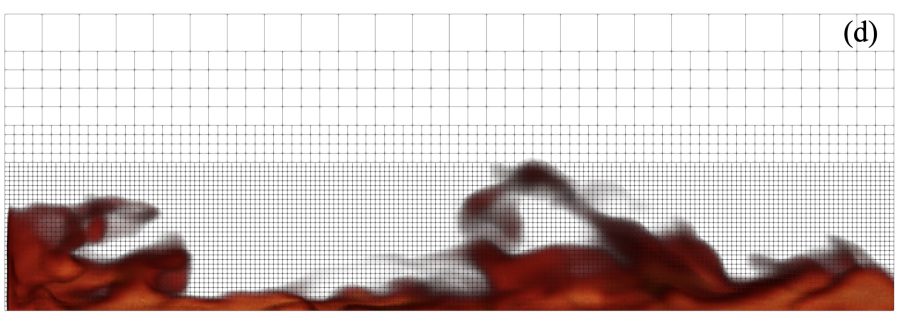

(e)

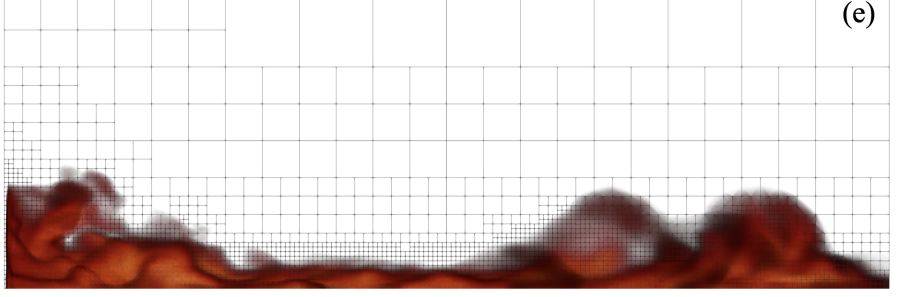

(f)

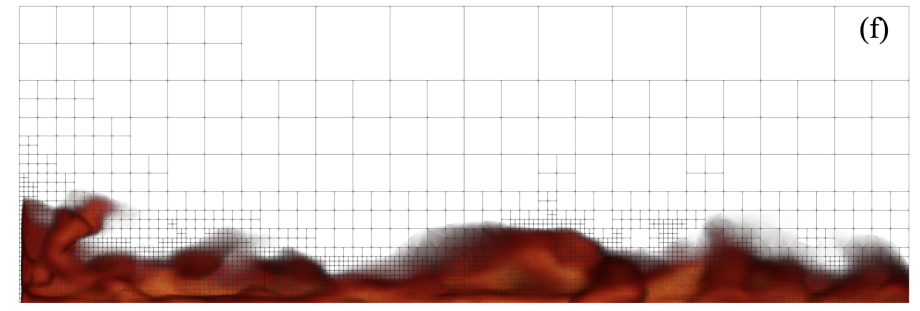

Figure 2. Visualization of static, AMR, and balanced AMR verification cases. Gas-phase combustion is visualized using volume renders of regions with high HRR for static $(\mathrm{a}, \mathrm{d})$, for AMR without load balancing $(\mathrm{b}, \mathrm{e})$, and AMR with load balancing (c,f) vertical panel spread cases at times $1.0 \mathrm{~s}(\mathrm{a}, \mathrm{b}, \mathrm{c})$ and $8.0 \mathrm{~s}(\mathrm{~d}, \mathrm{e}, \mathrm{f})$. Regions are colored by temperature from low (black) to high (yellow). The computational meshes are visualized in a plane through the center of the domain in each panel.

In total, the static mesh for the gaseous region was composed of 449,148 cells and the solid region was composed of 92,160 cells. We ran the simulation in parallel on 6 processors for $15 \mathrm{~s}$ of physical time.

\subsection{AMR simulation}

The physical configuration of the AMR simulation is identical to that for the static case, described in the previous section and shown in Fig. 1. A base mesh of $20 \mathrm{~cm}$ is created and the boundary faces along the gas-solid interface and burner are pre-refined by four levels to $1.25 \mathrm{~cm}$ resolution, for an initial cell count of 69,048 . The solid region is created as before and is therefore identical between the static mesh and AMR simulations.

Within the AMR simulation, four levels of refinement are used in the gaseous region to achieve $1.25 \mathrm{~cm}$ resolution, refining on gas-phase heat release rate (HRR) as a measure of combustion strength and location (top 99.9\% of values) and gradients in HRR (top 99.9\% of values) to fully encapsulate regions with combustion. The mesh is updated every four timesteps, and four buffer layers are used. Refinement fields are scaled at run-time to more easily allow assignment of lower and upper refinement bounds. It should be noted that other settings (e.g., other fields and refinement criteria) may give even better results (i.e., further decrease computational cost without affecting simulation accuracy) and the setup used here should be taken as illustrative rather than optimal.

Using the same physical configuration and mesh update procedure, two AMR simulations were performed, corresponding to static and dynamic approaches to grid decomposition and allocation to computational cores. The first AMR case used a time-invariant, geometric decomposition of the gaseous domain, while the second case used run-time load redistribution with the "scotch" decomposition in OpenFOAM. In each case, the decomposition of the solid region did not change with time. For the case with load balancing, mesh redistribution to computational cores was allowed every $0.1 \mathrm{~s}$ of simulation time. 


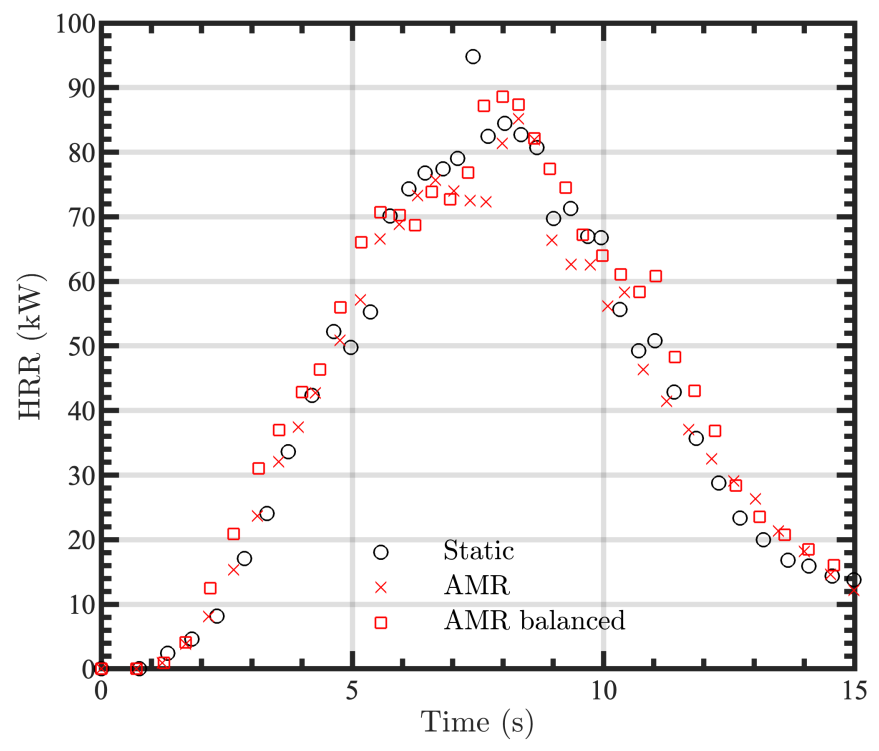

Figure 3. Comparison of chemical HRR time histories for the single panel vertical fire spread verification test case static (black circles), AMR without load balancing (red crosses), and AMR with load balancing (red squares) simulations. HRR is computed from instantaneous mass loss rates from the solid.

\subsection{Results and discussion}

Instantaneous snapshots of the flame spread up the panel are shown in Fig. 2 for the AMR and static simulations at 1.0, and $8.0 \mathrm{~s}$. In general, the flame structure is qualitatively similar between all three simulations (i.e., the static mesh simulation and the AMR simulations with and without load balancing), with the propagation of the flame up the panel occurring at roughly the same rate in all cases.

The resulting AMR meshes at 1.0, and $8.0 \mathrm{~s}$ are also shown in Fig. 2, illustrating the dynamically varying nature of the mesh and the gradually increasing number of grid cells in the AMR cases as the simulation proceeds. The AMR cases started with approximately 69,000 cells in the gaseous region and, once spun up, fluctuated around 115,000 cells (the static mesh simulation had roughly 449,000 cells). The baseline and balanced AMR simulations required an average of 6.8 and $5.4 \mathrm{cpu}$-hours per physical second of simulation time, respectively, computed as the product of the computation time and the number of cores divided by the physical duration of the simulation (i.e., $15 \mathrm{~s}$ ). Consequently, we were able to decrease the computational cost relative to the static case, which required an average of 13.6-cpu-hours per second of simulation time. Additionally, we have shown that, although the addition of load balancing makes the simulation even more computationally efficient, both AMR cases were significantly faster than their static counterpart.

From a quantitative perspective, the time history of volume-integrated chemical HRR is compared for the static and AMR simulations in Fig. 3. There is generally good agreement between the three simulations; it is emphasized, however, that the flame spread simulations are turbulent and some variability in the exact HRR time series is expected, independent of the mesh treatment. Nevertheless, the overall shapes of the time series are very similar between the three cases. We note that, although the turbulent nature of these simulations is expected to create local and instantaneous differences in the simulation results, the general correspondence between the AMR and static simulations in Fig. 3 suggests that the AMR simulations adequately capture the flame dynamics.

Finally, Fig. 4 shows instantaneous temperature profiles from the AMR and static simulations at $8 \mathrm{~s}$ (when HRR is close to its maximum) near the gas-solid interface at various heights above the burner. Once again, the temperatures are similar between the three simulations, and only small deviations between the results are observed. The 

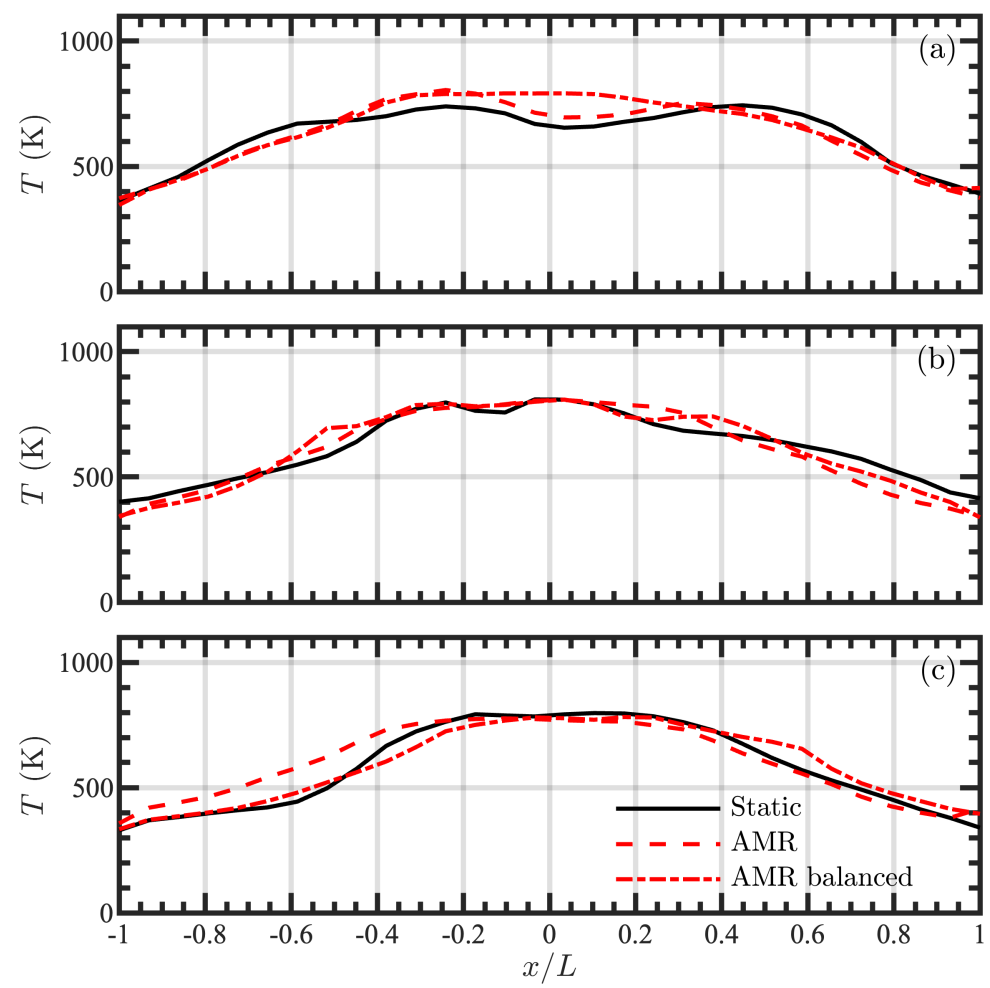

Figure 4. Instantaneous gas-phase temperature profiles near the time of peak HRR ( $8 \mathrm{~s})$ close to the gas-solid interface of the single panel vertical fire spread verification test case. Panels from top to bottom show profiles at panel heights of $z / H=0.2,0.4$, and 0.6 , where $H=2.4 \mathrm{~m}$ is the total height. Results are shown for static (solid black lines), AMR without load balancing (dashed red lines), and AMR with load balancing (dash-dot red lines) simulations. The transverse location $x$ is normalized by the half panel width $L=0.3 \mathrm{~m}$.

turbulent nature of these simulations also is expected to create local and instantaneous differences in the simulation results, and the overall correspondence between the AMR and static simulations in Fig. 4 suggests that the AMR simulations are able to accurately capture the flame dynamics.

It is important to note that the computational savings of the AMR simulation are dependent on the choice of refinement fields and their bounds. We have shown here that, for the choices outlined in Section 3.2, the AMR simulation provides results that are comparable to the static mesh results, at a substantially lower computational cost. Additional improvements in efficiency may be achievable for other choices of refinement fields and AMR thresholds, although it should also be noted that further improvements in static mesh simulation cost may also be possible. As such, the present results should be taken as illustrative of the accuracy and efficiency of fireDyMFoam, and greater (or lesser) gains may be realized in other problems.

\section{Validation: Two-panel vertical fire spread}

Building on the previous single panel vertical spread verification case, we now validate fireDyMFoam against experimental data for a panel spread case similar to the first verification case. In particular, a second panel with identical dimensions, opposite the first, was added to fully replicate the configuration in Chaos et al. [43]. For this configuration, two $[0.6 \times 2.4 \times 0.0154] \mathrm{m}$ corrugated cardboard panels are separated by a $[0.3 \times 0.6] \mathrm{m}$ 60-kW propane burner. Experimental and computational data for chemical HRR and gas-solid interface temperature and heat flux were computed by Chaos et al. [43]. Here, we focus on HRR as a global metric of importance for combustion problems, and only perform an AMR simulation. 


\subsection{AMR simulation}

The physical configuration of the two-panel case largely mimics that of the previous single-panel case shown in Fig. 1, but now also includes a second solid region opposite the first. As with the single panel verification case, the mesh boundaries adjacent to the panels on the gas-solid interface are pre-refined, for an initial cell count of 14,712 cells, before meshing the solid region. The solid-phase computational domain is created as described previously, resulting in 4,608 independent, 1D regions, each 10 cells in depth, for a total of 46, 080 cells. The case was run on a single processor for $150 \mathrm{~s}$; because the AMR simulation was run on only one processor, load balancing was not necessary for this case.

The physical models used in the gas phase are unchanged from the single panel spread verification case. Solid phase thermophysical properties are set following optimized values for the "triple-wall" corrugated cardboard reported in Chaos et al. [43]. The gas and solid phases are coupled as in the previous panel spread case with identical gas-solid interactions.

Following Chaos et al. [43], the gas-phase computational domain measures [0.3 $\times 8 \times$ 3] $\mathrm{m}$. Based on the recent success by Ren and Wang [44] in simulating a similar case at coarse resolution, here we use a base resolution of $10 \mathrm{~cm}$ and two levels of refinement to achieve an effective resolution of $2.5 \mathrm{~cm}$. The mesh was updated every other timestep; refinement fields and bounds were, however, not changed from those used in the singlepanel verification case.

\subsection{Results and discussion}

Experimental [43] and simulated chemical HRR (computed using the heat of combustion for pyrolysate from Ren et al. [29] and [45]) are compared in Fig. 5 for both the static mesh simulation from Chaos et al. [43] and the present AMR simulation using fireDyMFoam. After spinup of the AMR simulation, a maximum cell count of roughly 50,000 cells at peak HRR was required.

Quantitatively, Fig. 5 shows that the AMR simulation provides an accurate prediction of the exponential increase in HRR (the primary goal of this simulation), matching the experimental and computational results from Chaos et al. [43] reasonably well. The value of peak HRR is also similar between the experiments and AMR simulations. However, the time of peak HRR in the AMR simulation is slightly delayed relative to the experiments, and there is a faster decay of the HRR after the peak. Similar results were also obtained by Chaos et al. [43] using static mesh simulations, as shown in Fig. 5. As such, the difference in the decay rates is likely due to the physical modeling used in the simulations, rather than due to the use of AMR itself. For example, carbon oxidation has been identified by Chaos et al. [43] as a possible source of the discrepancies with the experiments, as it is not included in the pyrolysis model. Although model calibration is beyond the scope of the present study, Nyazika et al. [9] have reviewed the utility of such approaches for the case studied by Chaos et al. [43], where char oxidation was also mentioned as a potential source of error.

In the future, this case could be improved, for example, by re-tuning solid-phase thermophysical parameters for use with fireDyMFoam to better match the experimental data after the time of peak heat release. In terms of modeling, possible improvements include the incorporation of a char oxidation model in the solid phase (e.g., as implemented by FM Global [17]) and recent developments in wall models for convective heat transfer (e.g., as described by Ren and Wang [44]) and updated solid-phase thermophysical parameters (e.g., those used by Ren et al. [29], Ren and Wang [44], and [45]). We anticipate that, given the results in Fig. 5, each of these improvements will improve the agreement between the simulation and experimental results, and that AMR can still be used to decrease the computational cost of future simulations without negatively impacting accuracy. Nevertheless, the present AMR results using fireDyMFoam agree at least as well with the experimental data as the prior static mesh simulations from Chaos et al. [43]. 


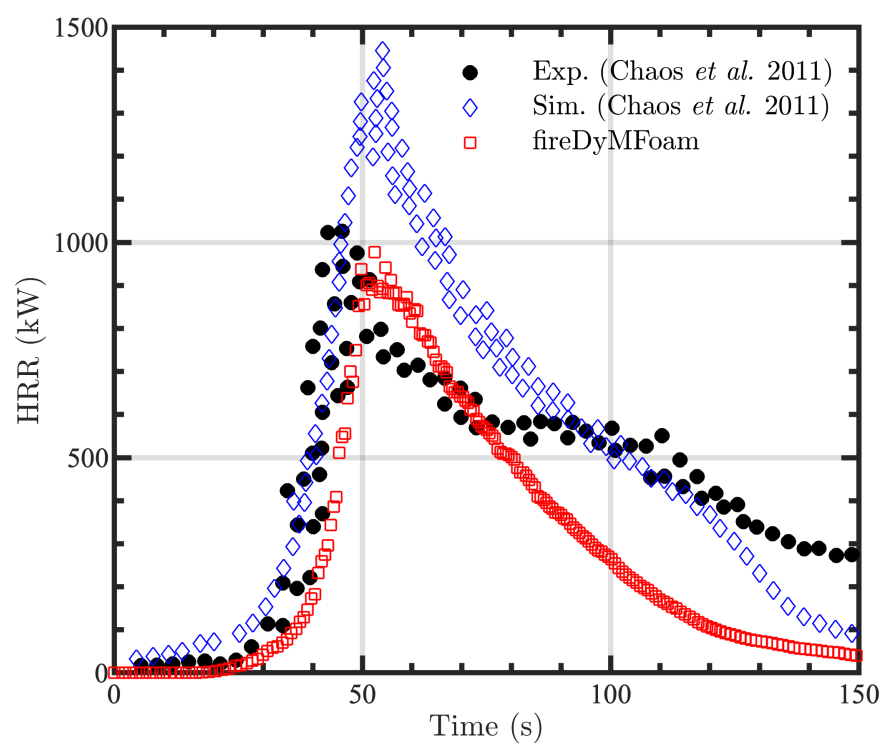

Figure 5. Time history of chemical heat release rate (HRR) for the two-panel fire spread validation case. Experimental and simulation results from Chaos et al. [43] are shown as black circles and blue diamonds, respectively, and AMR simulation results from fireDyMFoam are shown as red squares.

\section{Optimization: Fire suppression using liquid films and Lagrangian particles}

Having shown that AMR simulations using fireDyMFoam are computationally efficient and retain simulation fidelity, we now leverage the new solver for efficient optimizations of fire suppression methods, where the AMR simulations are coupled to the Dakota open-source toolkit [12]. For this demonstration, water is either introduced as a film at the top of the panel, or a sprinkler is used to spray water droplets at the panel.

Coupling between Dakota and OpenFOAM is based on previous similar studies [46-50] and uses a shell script to pass information between the two codes as follows:

1. Retrieve values (e.g., model parameters, boundary coefficients; initial or updated) from Dakota and convert to a format readable by OpenFOAM;

2. Edit OpenFOAM files with values from Dakota;

3. Run OpenFOAM case and post-process as necessary;

4. Compute cost function and pass to Dakota for updated parameter evaluation.

It should be noted that the coupling is quite general and treats the OpenFOAM simulations as a black box. This is beneficial because no modification of the computational software is required. There is a wide array of algorithms included with Dakota, ranging from local algorithms for gradient-based (e.g., Newton and conjugate-gradient methods [51,52]) and gradient-free (e.g., COBYLA [53]) optimization, to global methods such as genetic algorithms [12].

\subsection{Water film suppression}

The first demonstration case focuses on the suppression of vertical fire spread on a single panel via direct application of water. This case is based on the fireFoam tutorial flameSpreadWaterSuppresionPanel, as well as the fire spread simulations described in Sections 3 and 4 . For this case, a liquid water film is introduced at the top of the panel along the gas-solid interface to suppress fire spread. At the top of the panel, the water has a uniform velocity of $0.01 \mathrm{~m} / \mathrm{s}$ and a uniform temperature of $298.15 \mathrm{~K}$. The case is otherwise identical in physical configuration to the verification case discussed in Section 3.

Baseline cases with and without the water film were first simulated to demonstrate successful flame suppression. A base mesh with $20 \mathrm{~cm}$ resolution was created and boundary faces were refined to $2.5 \mathrm{~cm}$ resolution at the gas-solid interface and burner patches. Boundary faces were then extruded to create the solid region, as well as the liquid film 

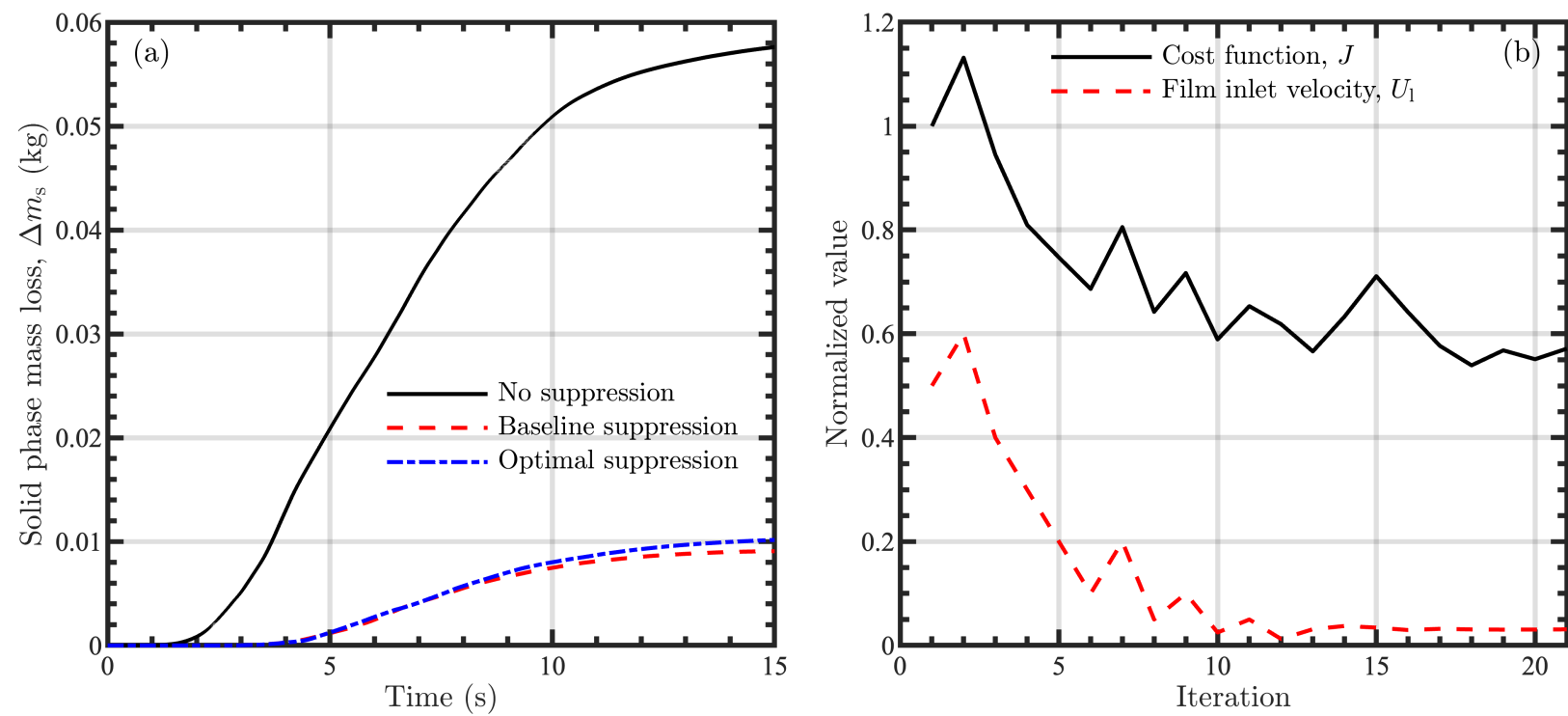

Figure 6. Solid-phase mass loss comparison for panel fire spread cases with and without water film suppression (a) and convergence history for the optimization (b). Panel (a) shows the mass loss for cases with no suppression (solid black line), baseline suppression parameters (dashed red line), and optimal suppression parameters (dash-dot blue line). Panel (b) shows normalized values of the cost function $J$ from Eq. (13) (solid black line) and the film inlet velocity $U_{1}$ (dashed red line).

region. Three levels of AMR were employed for an effective resolution of $2.5 \mathrm{~cm}$ in the gas phase. Refinement was based on regions of high HRR and the magnitude of its gradient. The solid and liquid phases were represented using static meshes with identical span-wise resolutions to that used in the gaseous domain. The film region was $1 \mathrm{~cm}$ and 1 cell thick, and the solid region was identical to the verification case in Section 3. Each simulation was run for $15 \mathrm{~s}$ of physical time.

A comparison of these cases is presented in Fig. 6(a), where time histories of solidphase mass loss are compared. Mass loss is used here as a surrogate for the extent of fire spread, where un-suppressed fires are expected to pyrolyze and burn more of the panel, resulting in greater mass loss. In Fig. 6(a), mass loss is shown to be substantially smaller at all times after roughly $2 \mathrm{~s}$ for the case with suppression, as compared to the case without suppression. Most importantly, the large magnitude of this difference suggests that it may be possible to reduce the amount of water introduced while still maintaining a substantially lower cumulative mass loss than the case without suppression.

The challenge of reducing the water flow rate while maintaining adequate flame suppression is ideally suited for optimization techniques. Recognizing that water conservation is important in water-scarce regions of the world, a cost function, $J$, can be constructed to take both solid-phase mass loss and water use into account as

$$
J=\alpha\left[\frac{\Delta m_{\mathrm{S}}}{\Delta m_{\mathrm{s}}(t=0)}\right]+\beta\left[\frac{U_{1}}{U_{1}(t=0)}\right],
$$

where $\Delta m_{\mathrm{S}}$ is the solid-phase change in mass, $U_{1}$ is the water injection velocity (a measure of water use), and $\alpha$ and $\beta$ are scaling coefficients set to 0.5 . Both terms in $J$ have been normalized by their respective initial values, with $\Delta m_{\mathrm{s}}(t=0)$ obtained from the baseline suppression case and $U_{1}(t=0)=0.01 \mathrm{~m} / \mathrm{s}$. Different values of $\alpha$ and $\beta$ result in different priorities given to mass loss and water usage during the optimization process, and here we simply set the two coefficients equal for demonstration purposes.

The COBYLA algorithm [53] was used to minimize $J$ subject to $0 \leq U_{1} \leq 0.02 \mathrm{~m} / \mathrm{s}$. These bounds were normalized to fall between 0 and 1 as $\hat{U}_{1}=\left(U_{1}-0\right) /(0.02-0)$ with a normalized starting point of $\hat{U}_{1}=0.5$. An optimal normalized water velocity of 


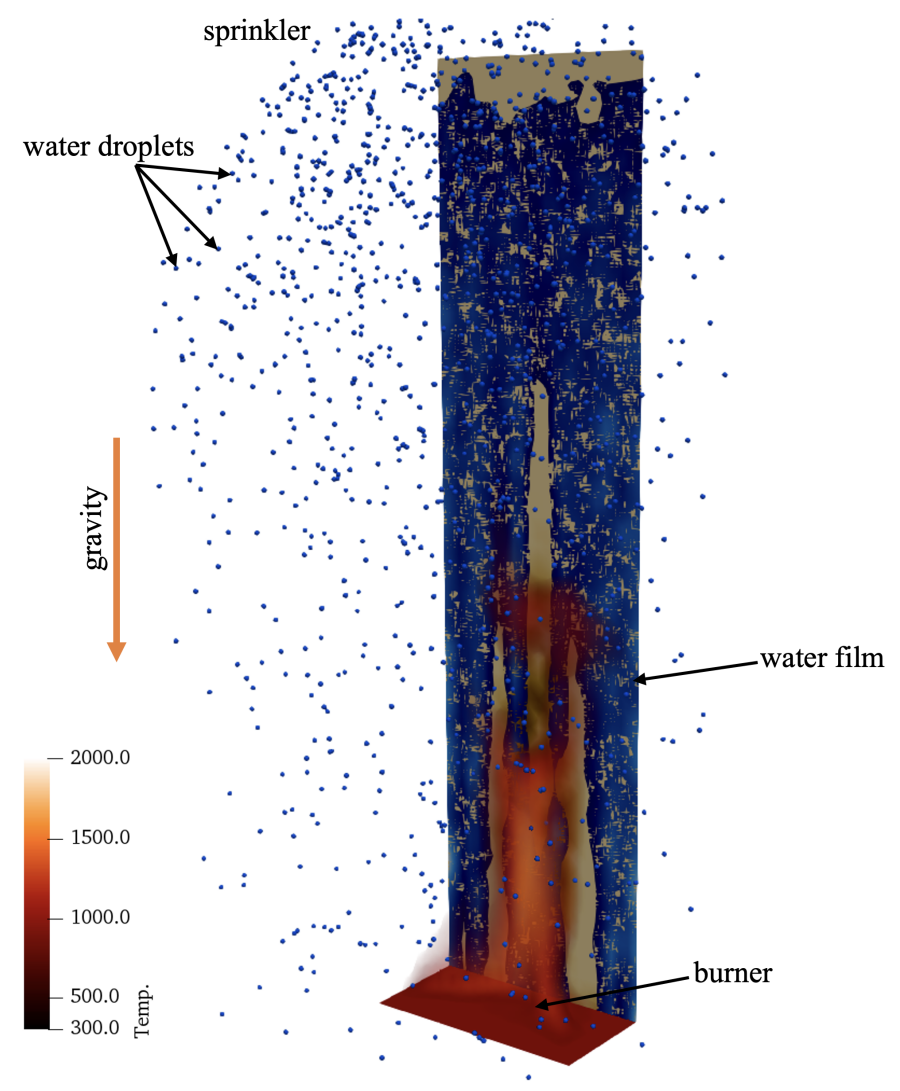

Figure 7. Visualization of baseline sprinkler case. Gas-phase combustion is visualized by a volumerender of cells with high temperature colored from low (black) to high (red) and solid-phase pyrolysis is visualized by char production colored low (tan) to high (black). Water is shown in blue for particleand film-based liquid.

approximately 0.03 , or $6 \times 10^{-4} \mathrm{~m} / \mathrm{s}$, was identified in 21 iterations and a plot of the convergence history is shown in Fig. 6(b).

To demonstrate the success of the optimization, the previous film simulation is updated to use the optimized inflow velocity. The mass loss time history is plotted against the original and baseline histories in Fig. 6(a), showing that we observe little difference between initial and optimized mass losses while decreasing the water inflow velocity by over an order of magnitude (corresponding to a relatively large amount of water savings), aligning nicely with our expectations for this case.

\subsection{Sprinkler suppression}

For the second optimization demonstration, a sprinkler is employed as the mode of water delivery. With the addition of Lagrangian particle tracking, this case incorporates all modeling functionality present in fireDyMFoam.

Instead of injecting a steady stream of water at the top of the panel, for this case a collection of water droplets (or "particles") is sprayed towards the panel at a fixed position, angle, mass flow rate, and temperature. A baseline case with particle settings based on similar tutorial cases (e.g., in The OpenFOAM Foundation [16] and FM Global [17]) is constructed with a sprinkler placed $0.5 \mathrm{~m}$ from the panel at a height of $2.4 \mathrm{~m}$ that is centered longitudinally. Water is sprayed downwards at an angle $45^{\circ}$ below the horizontal towards the panel at a rate of $0.1 \mathrm{~kg} / \mathrm{s}$, with a nominal exit speed of $3 \mathrm{~m} / \mathrm{s}$ and cone angle of $81^{\circ}$. There is two-way fluid-particle coupling and particle source terms are treated semi-implicitly in Eq. (1)-(4). Figure 7 shows an annotated 3D rendering of the baseline case. 
The baseline case was run for $15 \mathrm{~s}$ with the same physical and numerical settings as those used for the water film suppression test case described in Section 5.1 (with the addition of water droplets, as discussed above). Cumulative solid-phase mass loss is compared to the baseline case with suppression in Fig. 8(a) and is observed to be lower than the case without suppression. The baseline case is also visualized in Fig. 7, where we observe that some particles miss the panel entirely, suggesting that the injection cone angle as a parameter for optimization; a smaller angle would result in more water hitting the panel, increasing suppression effectiveness (as well as decreasing the volume of water necessary).

Once again, these results indicate that fire suppression comparable to that observed in the baseline case may be achievable using less water for a different choice of sprinkler parameters. Here we again consider a cost function that combines the solid-phase mass loss with total water consumption as

$$
J=\alpha\left[\frac{\Delta m_{\mathrm{s}}}{\Delta m_{\mathrm{s}}(t=0)}\right]+\beta\left[\frac{\dot{m}_{1}}{\dot{m}_{1}(t=0)}\right],
$$

where $\Delta m_{\mathrm{S}}$ is the solid-phase change in mass, $\dot{m}_{1}$ is total liquid mass introduced, and $\alpha$ and $\beta$ are scaling coefficients set to be 0.5 . Both terms have again been normalized by their respective values from the baseline simulation without suppression and maximum allowable mass introduction, respectively.

The cost function is minimized again using the COBYLA algorithm, varying injected mass flow rate, $\dot{m}_{1}$, and the injection cone angle, $\theta$, subject to the constraints $0 \leq \dot{m}_{1} \leq$ $0.2 \mathrm{~kg} / \mathrm{s}$ and $0^{\circ} \leq \theta \leq 90^{\circ}$, respectively. The baseline sprinkler case corresponds to a normalized starting point of $\hat{\dot{m}}_{1}=0.5$ and $\hat{\theta}=0.9$. In 40 iterations, optimal normalized values of mass flow rate and cone angle were determined to be approximately 0.05 and 0.155 , respectively, corresponding to $\dot{m}_{1}=0.01 \mathrm{~kg} / \mathrm{s}$ and $\theta=14.0^{\circ}$. Convergence history for this optimization is shown in Fig. 8(b).

The baseline sprinkler simulation is now updated using the optimized mass and cone angle values, with the time history of solid-phase mass loss shown in Fig. 8(a). Not only is the optimized solid-phase mass loss lower than the baseline case with suppression, the amount of water necessary was dramatically reduced. In particular, the water mass flow rate is reduced by an order of magnitude compared to the baseline case, which was made possible by reducing the cone angle by a factor of nearly six.

\section{Conclusions and future work}

We have developed a novel extension of the OpenFOAM solver fireFoam that incorporates $\mathrm{AMR}$ and retains all multiphase physics originally included with the solver. We have demonstrated that this solver, called fireDyMFoam, provides comparable agreement to static mesh simulations with the same fine-scale resolution for verification and validation cases, at substantially reduced computational cost. Additionally, the verification and validation spread cases were based on existing OpenFOAM tutorials for fireFoam to showcase the easy addition of AMR to fireFoam simulations. Although we primarily highlight the computational benefits of using AMR in this work, we also note that it relaxes hardware requirements; recognizing that simulations using AMR require fewer cells, they can be run on fewer processors (even serial runs are serviceable, as demonstrated by the optimization cases discussed previously).

We then applied fireDyMFoam inside an "outer-loop" optimization process to further demonstrate its potential. The Dakota toolkit was used to drive fireDyMFoam simulations of fire suppression, minimizing a cost function that balanced solid phase mass loss and water use. Although conceptually simple, these demonstrations were successful in finding non-trivial optima and the efficiency gains enabled by the solver will make future largescale studies more tractable.

There are a number of ways the solver could be improved. In the future, we plan to increase solid modeling capability, for example by incorporating high-fidelity pyrolysis 

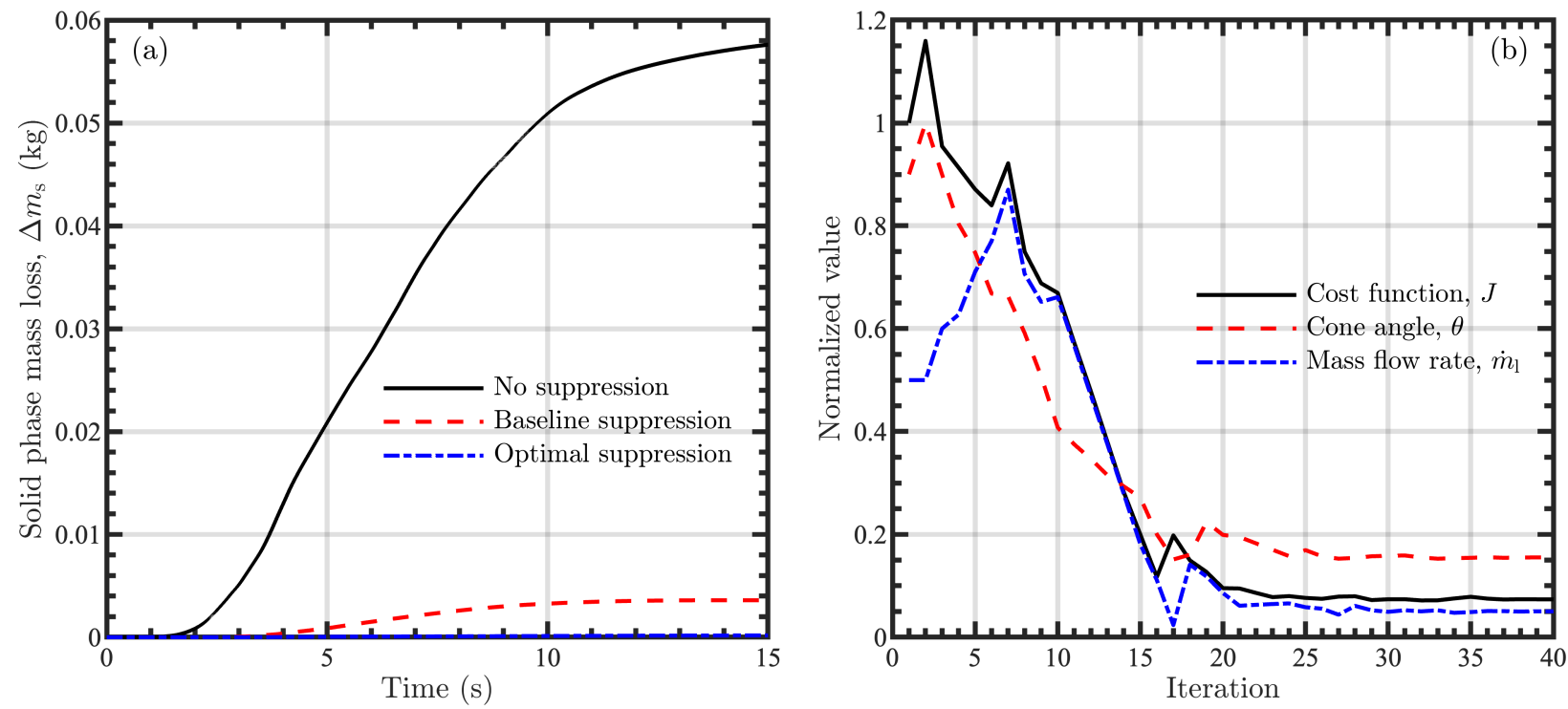

Figure 8. Solid-phase mass loss comparison for panel fire spread cases with and without water sprinkler suppression (a) and convergence history for the optimization (b). Panel (a) shows the mass loss for cases with no suppression (solid black line), baseline suppression parameters (dashed red line), and optimal suppression parameters (dash-dot blue line). Panel (b) shows normalized values of the cost function $J$ from Eq. (14) (solid black line), the cone angle $\theta$ (dashed red line), and the mass flow rate $\dot{m}_{1}$ (dash-dot blue line).

models in the Porous material Analysis Toolkit (PATO) [54]. As described previously, mapped patches must be pre-refined and protected from further refinement (thereby preventing the solid and liquid meshes from changing size) to enable the use of AMR within the current fireFoam modeling framework. Future work will address this first by adapting the solid solution procedure to be $3 \mathrm{D}$, thus allowing mesh changes similar to the AMR used in the gas-phase throughout this work and making large-scale fire spread problems more computationally tractable. Investigations of AMR use with respect to the liquid phase will then be warranted. It should be noted that the use of Lagrangian particles in the gas phase does not hinder the use of AMR, and other works (e.g., hyStrath [37]) have previously demonstrated such functionality.

Additionally, further investigation of AMR settings (e.g., refinement fields, frequency, the number of "buffer" layers) would be beneficial as well as the degree of refinement (i.e., the refinement level); noted previously, the chosen settings have been shown to work well but are not necessarily optimal. The coupling with Dakota may also be leveraged for efficient model calibration for fire spread and suppression simulations (similar to e.g., that used in Nigam [49] and Isaacs et al. [50]). An investigation of temporal, in addition to spatial, adaptivity will also be warranted as problem size increases.

Finally, future research directions also include extensions of the simulations presented in this work, including detailed pyrolysis and soot modeling and larger parameter spaces (e.g., solid fuel properties and more complex suppression configurations like those in Ren et al. [29]) for fire spread and suppression problems.

Author Contributions: Methodology, Gregory Rieker; Software, Caelan Lapointe, Nicholas Wimer, Sam Simons-Wellin, Jeffrey Glusman and Peter Hamlington; Writing - original draft, Caelan Lapointe; Writing - review editing, Nicholas Wimer, Sam Simons-Wellin, Jeffrey Glusman and Peter Hamlington.

Funding: CL was supported by the NSF Graduate Research Fellowship Program under award DGE 1144083. CL, NTW, SSW, JFG, GBR, and PEH were supported, in part, by SERDP under grant W912HQ-16-C-0026 and by the 3M Company under project MRA 19-08-0180 TO01. 
Data Availability Statement: The fireDyMFoam solver, associated libraries necessary for its compilation (e.g., a custom version of libdynamicFvMesh), and the verification and validation cases can be found at https://github.com/clapointe2011/public/of7/(link not yet active).

Acknowledgments: The authors would like to thank Yi Wang and Ning Ren of FM Global for their helpful input and for providing experimental data.

This work utilized the RMACC Summit supercomputer, which is supported by NSF (awards ACI-1532235 and ACI-1532236), the University of Colorado, Boulder, and Colorado State University.

Conflicts of Interest: The authors declare no conflict of interest. The funders had no role in the design of the study; in the collection, analyses, or interpretation of data; in the writing of the manuscript, or in the decision to publish the results.

\section{References}

1. Hanson, H.; Bradley, M.; Bossert, J.; Linn, R.; Younker, L. The potential and promise of physics-based wildfire simulation. Environmental Science $\mathcal{E}$ Policy 2000, 3, 161-172. doi: 10.1016/S1462-9011(00)00083-6.

2. Chen, Z.; Wen, J.; Xu, B.; Dembele, S. Extension of the eddy dissipation concept and smoke point soot model to the LES frame for fire simulations. Fire Safety Journal 2014, 64, 12-26.

3. Chen, Z.; Wen, J.; Xu, B.; Dembele, S. Large eddy simulation of a medium-scale methanol pool fire using the extended eddy dissipation concept. International Journal of Heat and Mass Transfer 2014, 70, 389-408.

4. Pope, S. Computationally efficient implementation of combustion chemistry using in situ adaptive tabulation. Combustion Theory and Modelling 1997, 1, 41-63.

5. Singer, M.; Pope, S.; Najm., H. Operator-splitting with ISAT to model reacting flow with detailed chemistry. Combustion Theory and Modelling 2006, 10, 199-217.

6. Singer, M.; Pope, S. Exploiting ISAT to solve the reaction-diffusion equation. Combustion Theory and Modelling 2004, 8, 361-383.

7. Niemeyer, K.; Sung, C.; Raju., M. Skeletal mechanism generation for surrogate fuels using directed relation graph with error propagation and sensitivity analysis. Combustion and Flame 2010, 157, 1760-1770.

8. Glusman, J.; Niemeyer, K.; Makowiecki, A.; Wimer, N.; Lapointe, C.; Rieker, G.; Hamlington, P.; Daily, J. Reduced Gas-Phase Kinetic Models for Burning of Douglas Fir. Frontiers in Mechanical Engineering 2019, 5, 40. doi:10.3389/fmech.2019.00040.

9. Nyazika, T.; Jimenez, M.; Samyn, F.; Bourbigot, S. Pyrolysis modeling, sensitivity analysis, and optimization techniques for combustible materials: A review. Journal of fire sciences 2019, 37, 377-433.

10. Lapointe, C.; Wimer, N.; Glusman, J.; Makowiecki, A.; Daily, J.; Rieker, G.; Hamlington, P. Efficient simulation of turbulent diffusion flames in OpenFOAM using adaptive mesh refinement. Fire Safety Journal 2020, 111, 102934.

11. Wang, Y.; Chatterjee, P.; de Ris, J. Large eddy simulation of fire plumes. Proceedings of the Combustion Institute 2011, 33, 2473-2480.

12. Adams, B.; Bohnhoff, W.; Dalbey, K.; Eddy, J.; Eldred, M.; Gay, D.; Haskell, K.; Hough, P.; Swiler, L. DAKOTA, a multilevel parallel object-oriented framework for design optimization, parameter estimation, uncertainty quantification, and sensitivity analysis: version 5.0 user's manual. Sandia National Laboratories, Tech. Rep. SAND2010-2183 2009.

13. Fire Dynamics Simulator User's Guide. NIST Special Publication, 1019.

14. Lee, J. Numerical analysis on the rapid fire suppression using a water mist nozzle in a fire compartment with a door opening. Nuclear Engineering and Technology 2019, 51, 410-423.

15. Wang, Z.; Wang, W.; Wang, Q. Optimization of water mist droplet size by using CFD modeling for fire suppressions. Journal of Loss Prevention in the Process Industries 2016, 44, 626-632.

16. The OpenFOAM Foundation. OpenFOAM Development Repository; Github, 2021.

17. FM Global. FireFOAM Development Repository; Github, 2020.

18. Maragkos, G.; Rauwoens, P.; Merci, B. Application of FDS and FireFOAM in Large Eddy Simulations of a Turbulent Buoyant Helium Plume. Combustion Science and Technology 2012, 184, 1108-1120.

19. Maragkos, G.; Rauwoens, P.; Wang, Y.; Merci, B. Large eddy simulations of the flow in the near-field region of a turbulent buoyant helium plume. Flow, Turbulence and Combustion 2013, $90,511-543$. 
20. Maragkos, G.; Merci, B. Large Eddy simulations of $\mathrm{CH} 4$ fire plumes. Flow, Turbulence and Combustion 2017, 99, 239-278.

21. Maragkos, G.; Beji, T.; Merci, B. Implementation and Evaluation of the Dynamic Smagorinsky Model and an Eddy Dissipation Model with Multiple Reaction Time Scales in FireFOAM 2017.

22. Vilfayeau, S.; Ren, N.; Wang, Y.; Trouvé, A. Numerical simulation of under-ventilated liquidfueled compartment fires with flame extinction and thermally-driven fuel evaporation. Proceedings of the Combustion Institute 2015, 35, 2563-2571.

23. Salmon, F.; Lacanette, D.; Mindeguia, J.; Sirieix, C.; Ferrier, C.; Leblanc, J. FireFOAM simulation of a localised fire in a gallery 2018. 1107, 042017.

24. Ding, Y.; Wang, C.; Lu, S. Modeling the pyrolysis of wet wood using FireFOAM. Energy Conversion and Management 2015, 98, 500-506.

25. Ding, Y.; Wang, C.; Lu, S. Large Eddy Simulation of Fire Spread. Procedia Engineering 2014, 71, 537-543.

26. Meredith, K.; de Vries, J.; Wang, Y.; Xin, Y. A comprehensive model for simulating the interaction of water with solid surfaces in fire suppression environments. Proceedings of the Combustion Institute 2013, 34, 2719-2726.

27. Fukumoto, K.; Wang, C.; Wen, J. Large eddy simulation of upward flame spread on PMMA walls with a fully coupled fluid-solid approach. Combustion and Flame 2018, 190, 365-387.

28. Trouvé, A.; Wang, Y. Large eddy simulation of compartment fires. International Journal of Computational Fluid Dynamics 2010, 24, 449-466.

29. Ren, N.; de Vries, J.; Zhou, X.; Chaos, M.; Meredith, K.; Wang, Y. Large-scale fire suppression modeling of corrugated cardboard boxes on wood pallets in rack-storage configurations. Fire Safety Journal 2017, 91, 695-704.

30. Meredith, K.; Xin, Y.; de Vries, J. A numerical model for simulation of thin-film water transport over solid fuel surfaces. Fire Safety Science 2011, 10, 415-428.

31. Meredith, K.; Heather, A.; De Vries, J.; Xin, Y. A numerical model for partially-wetted flow of thin liquid films. Computational Methods in Multiphase Flow VI 2011, 70, 239.

32. Chaos, M.; Khan, M.; Krishnamoorthy, N.; de Ris, J.; Dorofeev, S. Evaluation of optimization schemes and determination of solid fuel properties for CFD fire models using bench-scale pyrolysis tests. Proceedings of the Combustion Institute 2011, 33, 2599-2606.

33. Vinayak, A. Mathematical Modeling \& Simulation of Pyrolysis \& Flame Spread in OpenFOAM. Master's thesis, 2017.

34. Jasak, H.; Gosman, A.D. Automatic Resolution Control for the Finite-Volume Method, Part 2: Adaptive Mesh Refinement and Coarsening. Numerical Heat Transfer: Part B: Fundamentals 2000, $38,257-271$.

35. Zhang, W.; Almgren, A.; Beckner, V.; Bell, J.; Blaschke, J.; Chan, C.; Day, M.; Friesen, B.; Gott, K.; Graves, D.; Katz, M.; Myers, A.; Nguyen, T.; Nonaka, A.; Rosso, M.; Williams, S.; Zingale, M. AMReX: a framework for block-structured adaptive mesh refinement. Journal of Open Source Software 2019, 4, 1370. doi:10.21105/joss.01370.

36. Rettenmaier, D.; Deising, D.; Ouedraogo, Y.; Gjonaj, E.; De Gersem, H.; Bothe, D.; Tropea, C.; Marschall, H. Load balanced 2D and 3D adaptive mesh refinement in OpenFOAM. SoftwareX 2019, 10, 100317.

37. hyStrath Repository; Github, 2021.

38. The National Renewable Energy Lab. Simulator for Wind Farm Applications (SOWFA) Repository; Github, 2021.

39. Lab, U. Hybrid Central Solvers Repository; Github, 2021.

40. Yoshizawa, A. Statistical theory for compressible turbulent shear flows, with the application to subgrid modeling. The Physics of Fluids 1986, 29, 2152-2164.

41. Vilfayeau, S. Large eddy simulation of fire extinction phenomena. PhD thesis, University of Maryland, College Park, 2015.

42. Ren, N.; Wang, Y.; Vilfayeau, S.; Trouvé, A. Large eddy simulation of turbulent vertical wall fires supplied with gaseous fuel through porous burners. Combustion and Flame 2016, 169, 194-208.

43. Chaos, M.; Khan, M.; Krishnamoorthy, N.; Chatterjee, P.; Wang, Y.; Dorofeev, S. Experiments and modeling of single-and triple-wall corrugated cardboard: Effective material properties and fire behavior. Fire and Materials 2011, pp. 625-636.

44. Ren, N.; Wang, Y. A convective heat transfer model for LES fire modeling. Proceedings of the Combustion Institute 2020. 
45. Wang, Y.; Meredith, K.; Zhou, X.; Chatterjee, P.; Xin, Y.; Chaos, M.; Ren, N.; Dorofeev, S. Numerical simulation of sprinkler suppression of rack storage fires. Fire Safety Science 2014, $11,1170-1183$.

46. Jareteg, A. Coupling of Dakota and OpenFOAM for automatic parameterized optimization. Proceedings of CFD with OpenSource Software, 2013, Edited by H. Nilsson, 2013.

47. Holzmann, T. Optimization with Dakota; 2018.

48. Gurreroo, J. Design of experiments, space exploration, and numerical optimization using Dakota and OpenFOAM. Proceedings of the 11th OpenFOAM workshop, 2016.

49. Nigam, S. Large Eddy Simulations of Industrial Burners. Master's thesis, University of Colorado, Boulder, 2018.

50. Isaacs, S.; Lapointe, C.; Hamlington, P. Development and Application of a Thin Flat Heat Pipe Design Optimization Tool for Small Satellite Systems. Journal of Electronic Packaging 2020.

51. Fletcher, R.; Reeves, C.M. Function minimization by conjugate gradients. The Computer Journal 1964, 7, 149-154.

52. Fletcher, R. A new approach to variable metric algorithms. The Computer Journal 1970, 13, 317322.

53. Powell, M. A direct search optimization method that models the objective and constraint functions by linear interpolation. In Advances in optimization and numerical analysis; Springer, 1994; pp. 51-67.

54. Lachaud, J.; Scoggins, J.; Magin, T.; Meyer, M.; Mansour, N. A generic local thermal equilibrium model for porous reactive materials submitted to high temperatures. International Journal of Heat and Mass Transfer 2017, 108, 1406-1417. 
